CORRIGENDUM

Owing to an error, reference 7 was misplaced in the article by B. D. Grant and $A$. Audhya $(7,1151-1154 ; 2005)$. Its content is not in contrast with what was reported by Gallop and coworkers. The corrected text should read as follows:

\title{
Membrane curvature
}

A key biophysical event in membrane transport is the formation of highly curved membranes in the generation of vesicles or tubules. Recent work has uncovered new proteins that promote membrane curvature, thus providing insight into how this process occurs. Jennifer Gallop (Cambridge, UK) presented work conducted with Harvey McMahon and colleagues on the role of endophilin in endocytosis. She presented evidence that the BAR domain of endophilin lacks intrinsic lysophosphatitic-acid-acyltransferase activity, arguing against the findings of others ${ }^{8}$. Her crystalstructure data indicates that endophilin BAR-domain dimers form a concave membrane-binding interface, consistent with proposals that endophilin drives positive membrane curvature ${ }^{7,0}$.

This has now been corrected online.

7. Farsad, K. et al. J. CellBiol. 155, 193-200 (2001).

8. Schmidt, A. et al. Noture 401, 133-141 (1999).

9. Gallop, J. L. \& McMahon, H. T. Biachem. Soc. Symp. 223-431 (2005). 